\title{
REPRESENTATION OF A $p$-HARMONIC FUNCTION NEAR AN ISOLATED SINGULARITY IN THE PLANE
}

\author{
Ulf Janfalk \\ Linköping University, Department of Mathematics, \\ S-581 83 Linköping, Sweden; uljan@math.liu.se
}

\begin{abstract}
A representation theorem is proved for a $p$-harmonic function $(1<p<\infty)$ near an isolated singularity in the plane. The proof uses stream functions and the hodograph method. The singularities can be classified as removable, poles and essential as is the case for analytic functions. Via the representation we obtain a complete classification of isolated singularities, analogous to the classical one for harmonic functions, in terms of the growth of the function near the singularity. In the case $p=2$ this reduces to the classical one. Further, we derive some properties of the stream function and singular expansions of both the $p$-harmonic function and its stream function in the case of a pole.
\end{abstract}

\section{Introduction}

Let $\varphi$ be a $p$-harmonic function in the domain $\Omega=\{z \in \mathbf{C}: 0<|z|<1\}$, i.e. a weak solution of the equation

$$
\operatorname{div}\left(|\nabla \varphi|^{p-2} \nabla \varphi\right)=0
$$

in the domain $\Omega \subset \mathbf{C}$. The purpose of this paper is to derive a representation formula for $\varphi$, valid in a punctured neighbourhood of $z=0$, where $\varphi$ is assumed to have a non-removable singularity of the type pole, defined below.

The proof is based on the hodograph method and the fact that $\varphi$ has a $p^{\prime}$-harmonic $\left((1 / p)+\left(1 / p^{\prime}\right)=1\right)$ stream function, denoted $\psi$, in every simply connected subdomain of $\Omega$. Some consequences of the representation theorem are derived:

1. Necessary and sufficient conditions for $\psi$ to be defined and $C^{\infty}$ in $\Omega$.

2. A representation formula for $\psi$ of the same type as that for $\varphi$.

3. Classification of all possible types of singularities.

4. Singular expansions for $\varphi$ and $\psi$ near $z=0$.

The representation has the form of linear superposition

$$
\left(\begin{array}{c}
z \\
\varphi
\end{array}\right)=\sum_{m=-N+1}^{\infty} A_{m}\left(\begin{array}{l}
Z_{m}(w) \\
\Phi_{m}(w)
\end{array}\right)
$$

1991 Mathematics Subject Classification: Primary 35J; Secondary 35C. 
where $w=\left(\sqrt[N]{\varphi_{x}-i \varphi_{y}}\right)^{-1}$. When considered alone, each pair $\left(\begin{array}{c}Z_{m}(w) \\ \Phi_{m}(w)\end{array}\right)$ locally generates a quasi-radial $p$-harmonic function for $w \neq 0$.

In $[\mathrm{M}]$, J. Manfredi treats the same problem using a different variant of the hodograph method. There the main attention is focused on analyzing the properties of the mapping $z(w)$ from which the information about $\varphi$ is extracted. A representation of $z(w)$ identical to the one given here, is obtained in Theorem 3 and a singular expansion of the same form as here is given in Theorem 2. The results regarding the singular expansion are the same in the cases $N \geq 3$ and $N=1, p>2$. In the remaining cases the results here go farther.

\section{The setup}

2.1. Background. Put $\Omega=\{z \in \mathbf{C}: 0<|z|<1\}$, and let $1<p<\infty$. Suppose $\varphi$ is $p$-harmonic in $\Omega$, i.e. $\varphi \in W_{\text {loc }}^{1, p}(\Omega)$ and

$$
\int_{\Omega}|\nabla \varphi|^{p-2} \nabla \varphi \cdot \nabla \eta d x=0
$$

for all $\eta \in C_{0}^{1}(\Omega)$.

Then it is well known that $\varphi \in C_{\text {loc }}^{1, \alpha}$ (see [L1]) and $\varphi$ is real analytic in every subdomain of $\Omega$ where $\nabla \varphi \neq 0$ (see [L1] p. 208 and [H]). Let $D \subset \mathbf{C}$ be an open and connected set.

Definition 1. A function $f: D \rightarrow \mathbf{C}$ is said to be $K$-quasi-regular if 1. $f \in W_{\text {loc }}^{1,2}(D)$,

2. $\left|f_{\bar{z}}\right| \leq(K-1)\left|f_{z}\right| /(K+1)$ a.e in $D$ for some $K \geq 1$.

From [B-I] we have that the complex gradient $\varphi_{x}-i \varphi_{y}$ is $K$-quasi-regular, $K \geq 1$. It is a well known fact that $K$-quasi-regular mappings are continuous. Further, the complex gradient has a representation

$$
\varphi_{x}-i \varphi_{y}=h \circ \chi
$$

where $\chi$ is $K$-quasi-conformal and $h$ is analytic (see [R] Theorem 2.17, p. 45).

Let $\chi: \Omega \rightarrow \Omega^{\prime}$ and $h: \Omega^{\prime} \rightarrow \mathbf{C}$ be such that $\varphi_{x}-i \varphi_{y}=h \circ \chi$. From a theorem on removable sets for quasi-conformal mappings (see [V], p. 52) we conclude that isolated singularities are removable, i.e. $\chi$ is quasi-conformal in $\Omega \cup\{0\}$. By composing $\chi$ with a conformal mapping we can assume that $\chi(0)=0$. The representation of $\varphi_{x}-i \varphi_{y}$ is not unique, but all relevant properties, such as the order of poles and zeros (see $[R]$, p. 66) are independent of the choice of representation. This leads to the following definition.

Definition 2. Suppose $h$ has a pole of order $N$ at $\chi\left(z_{0}\right)$. Then $\varphi$ is said to have a pole of order $N-1$ at $z_{0}$. 
A pole of order zero for $\varphi$ will sometimes be called a singularity of fundamental solution type, since the asymptotic behaviour will resemble that of the fundamental solution $r^{(p-2) /(p-1)}$.

In this paper we will treat the case where $h$ has a pole of order $N \geq 1$.

Lemma 1. There exists $r: 0<r \leq 1$ such that

$$
\frac{\partial\left(\varphi_{x}, \varphi_{y}\right)}{\partial(x, y)}=\left|\frac{\partial}{\partial \bar{z}} h \circ \chi\right|^{2}-\left|\frac{\partial}{\partial z} h \circ \chi\right|^{2}<0 \quad \text { for } 0<|z|<r .
$$

Proof. Since $h$ has a pole at $\xi=0$ there exists a punctured neighbourhood $U$ of $\xi=0$ such that

$$
\inf _{\xi \in U}\left(|h(\xi)|,\left|h^{\prime}(\xi)\right|\right)>0 .
$$

Thus there exists a punctured neighbourhood of $z=0$ in which $\nabla \varphi$ is non-zero. Consequently, $\varphi$ is real analytic there and we get

$$
\chi \in C(\{z:|z|<r\}) \cap C^{\infty}(\{z: 0<|z|<r\})
$$

for some $r>0$. By [A-L, Theorem 3, p. 161], there is a set $E$ of isolated points in $\Omega$ such that

$$
\frac{\partial\left(\varphi_{x}, \varphi_{y}\right)}{\partial(x, y)}<0 \quad \text { in } \Omega \backslash E .
$$

By the proof of that theorem we have that $E=S \cup T$ where

$$
S=\{z \in \Omega: h \circ \chi(z)=0\}, \quad T=\left\{z \in \Omega: h^{\prime} \circ \chi(z)=0\right\} .
$$

By continuity of $\chi$ there is an $r>0$ such that $\chi(\{0<|z|<r\}) \subset U \cap \chi(\Omega)$ and we get $\{0<|z|<r\} \cap(S \cup T)=\emptyset$, i.e.

$$
\frac{\partial\left(\varphi_{x}, \varphi_{y}\right)}{\partial(x, y)}<0 \quad \text { for } 0<|z|<r
$$

Corollary. We have

$$
\frac{\partial}{\partial z} \chi(z) \neq 0
$$

for $0<|z|<r$.

Proof. By the definition of quasi-conformal mappings

$$
\left|\chi_{\bar{z}}\right| \leq \frac{K-1}{K+1}\left|\chi_{z}\right|
$$

a.e. in $\Omega \cup\{0\}$ for some $K \geq 1$. Since $\chi$ turned out to be smooth for $0<|z|<r$, this can now be interpreted pointwise. We have $\frac{\partial\left(\varphi_{x}, \varphi_{y}\right)}{\partial(x, y)}=\left|\frac{\partial}{\partial \bar{z}} h \circ \chi\right|^{2}-\left|\frac{\partial}{\partial z} h \circ \chi\right|^{2}=\left|h^{\prime}(\chi(z))\right|^{2}\left(\left|\chi_{\bar{z}}\right|^{2}-\left|\chi_{z}\right|^{2}\right)<0, \quad 0<|z|<r$.

Hence $\left|\chi_{z}(z)\right| \neq 0$. 
If we for a moment regard

$$
\left\{\begin{array}{l}
u(x, y)=\operatorname{Re} \chi(z) \\
v(x, y)=\operatorname{Im} \chi(z)
\end{array}\right.
$$

as an ordinary change of coordinates in $\mathbf{R}^{2}$, we get $(z=x+i y)$

$$
\frac{\partial(u, v)}{\partial(x, y)}=\left|\chi_{z}(x, y)\right|^{2}-\left|\chi_{\bar{z}}(x, y)\right|^{2}>0, \quad 0<|z|<r .
$$

From the inverse function theorem we get $\chi^{-1} \in C^{1}(\chi(\{0<|z|<r\}))$. It then follows from the chain rule that $\chi^{-1} \in C^{\infty}(\chi(\{0<|z|<r\}))$.

2.2. Construction of the desired mappings. Take $r$ as in Lemma 1 and put $\Omega_{r}=\{z: 0<|z|<r\}$. Then $\nabla \varphi$ and $\left(\partial\left(\varphi_{x}, \varphi_{y}\right)\right) /(\partial(x, y))$ are both nonzero in $\Omega_{r}, \chi \in C\left(\Omega_{r} \cup\{0\}\right) \cap C^{\infty}\left(\Omega_{r}\right)$ and $\chi^{-1} \in C\left(\chi\left(\Omega_{r} \cup\{0\}\right)\right) \cap C^{\infty}\left(\chi\left(\Omega_{r}\right)\right)$. The following construction is illustrated by Figure 1 .

Put $\xi=\chi(z), \zeta^{\prime}=\varphi_{x}+i \varphi_{y}=\bar{h} \circ \chi(z)$ and let $N \geq 1$ be the order of the pole of $h$. Let $\delta>0$ and put $U_{\delta}=\{\xi: 0<|\xi|<\delta\}$. If $\delta$ is sufficiently small there is a univalent analytic function $g$ defined on $U_{\delta}$, having a simple pole at $\xi=0$ such that $h(\xi)=(g(\xi))^{N}$ for all $\xi \in U_{\delta}$.

Put $\zeta=\bar{g}(\xi)$ and put $\omega\left(q_{0}\right)=\left\{\zeta:|\zeta|>q_{0}\right\}$. Choose $\delta>0$ so small that $\chi\left(\Omega_{r}\right) \supset U_{\delta}$ and choose $q_{0}$ large enough such that $\overline{\omega\left(q_{0}\right)} \subset \bar{g}\left(U_{\delta}\right)$.

Put $\Omega_{0}=\left(\chi^{-1} \circ(\bar{g})^{-1}\right)\left(\omega\left(q_{0}\right)\right)$. Then we get $\Omega_{0} \subset \Omega_{r}$ and $\left(\varphi_{x}+i \varphi_{y}\right)(z)$ $=((\bar{g} \circ \chi)(z))^{N}$ for $z \in \Omega_{0}$. Hence, $\zeta^{\prime}=(\zeta)^{N}$ and that mapping maps $\omega\left(q_{0}\right)$ onto the set $\omega^{\prime}\left(q_{0}^{\prime}\right)=\left\{\zeta^{\prime}:\left|\zeta^{\prime}\right|>q_{0}^{\prime}\right\}$ where $q_{0}^{\prime}=\left(q_{0}\right)^{N}$.

By the preceding discussion $z(\zeta)=\chi^{-1} \circ(\bar{g})^{-1}(\zeta)$ becomes a smooth homeomorphism from $\omega\left(q_{0}\right)$ to $\Omega_{0}$ and $\lim _{|\zeta| \rightarrow \infty} z(\zeta)=0$. The obvious converse holds for the inverse, $\zeta(z)$. Hence, $\varphi(\zeta) \in C^{\infty}\left(\omega\left(q_{0}\right)\right)$, i.e. this change of coordinates does not create any new singularities.

Later we will also use $w=\varrho e^{i \theta}=(\bar{\zeta})^{-1}$ instead of $\zeta$ (see Figure 1).

2.3. The hodograph transformation. Since $z(\zeta)$ is smooth it follows that $\partial \bar{\Omega}_{0}$ is smooth. Take an arbitrary point on $\partial \bar{\Omega}_{0}$. Let $\Gamma$ be an arbitrary simple curve joining $z=0$ and the point on $\partial \bar{\Omega}_{0}$. Put $\widetilde{\Omega}=\Omega_{0} \backslash \Gamma$. $\widetilde{\Omega}$ is then simply connected since its complement is connected with respect to the extended plane. $\varphi$ is still $p$-harmonic in $\widetilde{\Omega}$ and from Lemma 1 we know that $\nabla \varphi$ and $\left(\partial\left(\varphi_{x}, \varphi_{y}\right)\right) /(\partial(x, y))$ are non-zero in $\widetilde{\Omega}$.

By [Ar2, Theorem 1, p. 75], we obtain that $\varphi$ has a $p^{\prime}$-harmonic stream function $\psi$ in $\widetilde{\Omega}$ such that

$$
\psi_{x}=-|\nabla \varphi|^{p-2} \varphi_{y} \quad \psi_{y}=|\nabla \varphi|^{p-2} \varphi_{x} .
$$




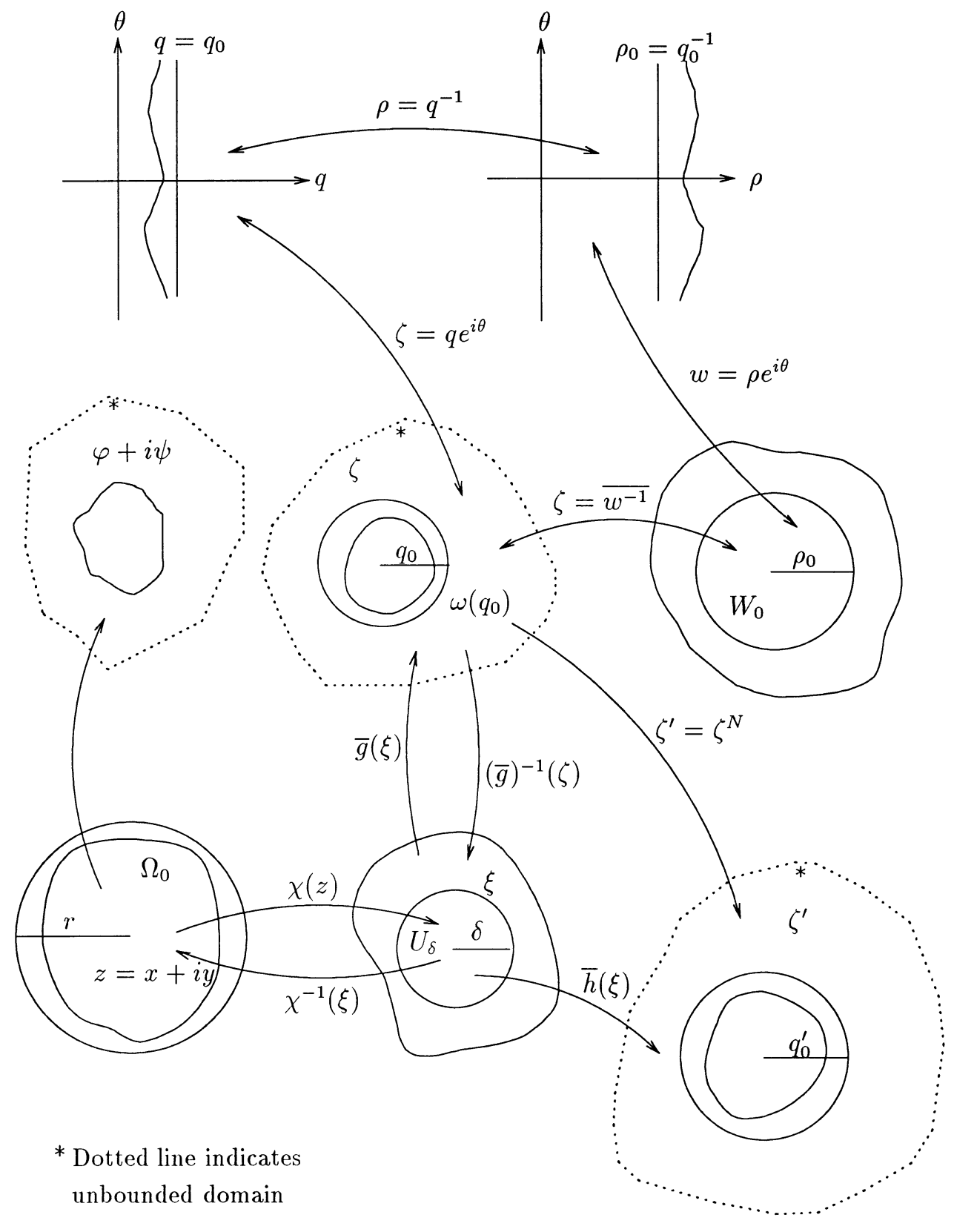

Figure 1. Mapping map 
The equations (2) are the so called $p$-Cauchy-Riemann equations. Put $q^{\prime} e^{i \theta^{\prime}}=\zeta^{\prime}$. Then $(\partial(\varphi, \psi)) /(\partial(x, y))=|\nabla \varphi|^{p}=\left(q^{\prime}\right)^{p}>0$ in $\widetilde{\Omega}$.

From the preceding mapping constructions we infer that $z=z(\zeta)$ and $\varphi=$ $\varphi(\zeta)$ are well defined and smooth in $\overline{\omega\left(q_{0}\right)}$.

Let $\Gamma$ and $\widetilde{\Omega}$ be as above. Let $\Gamma^{\prime}$ be the image of $\Gamma$ in the $\zeta$-plane. Now, $\widetilde{\Omega}$ and $\omega\left(q_{0}\right) \backslash \Gamma^{\prime}$ are in one-to-one correspondence by construction and $\psi$ can be defined in $\omega\left(q_{0}\right) \backslash \Gamma^{\prime}$. Let $\zeta_{0} \in \omega\left(q_{0}\right) \backslash \Gamma^{\prime}$. There is a neighbourhood $V$ of $\zeta_{0}$ and a neighbourhood $V^{\prime}$ of $\zeta_{0}^{\prime}$ such that the mapping $\zeta \rightarrow(\zeta)^{N}=\zeta^{\prime}$ can be defined uniquely as a smooth bijection of $V$ onto $V^{\prime}$.

$z=z\left(\zeta^{\prime}\right)$ then becomes well-defined and smooth near $\zeta_{0}^{\prime}=\left(\zeta_{0}\right)^{N}$. We can therefore use the hodograph method in the same way as in [Ar1, p. 90]. There it is shown that $\varphi$ and $\psi$ locally satisfy the following Chaplygin-type system

$$
\varphi_{\theta^{\prime}}=\frac{\psi_{q^{\prime}}}{\left(q^{\prime}\right)^{p-3}}, \quad \varphi_{q^{\prime}}=\frac{(1-p) \psi_{\theta^{\prime}}}{\left(q^{\prime}\right)^{p-1}} .
$$

If we put $\mu=-\log q$ we get $q^{\prime}=e^{-N \mu}$ and $\theta^{\prime}=N \theta$. This gives the following modified version of the Chaplygin system (3):

$$
\varphi_{\mu}=(p-1) e^{N(p-2) \mu} \psi_{\theta}, \quad \varphi_{\theta}=-e^{N(p-2) \mu} \psi_{\mu} .
$$

Cross-differentiation and elimination yields:

$$
\varphi_{\theta \theta}+\frac{1}{p-1} \varphi_{\mu \mu}-N \frac{p-2}{p-1} \varphi_{\mu}=0, \quad \psi_{\theta \theta}+\frac{1}{p-1} \psi_{\mu \mu}+N \frac{p-2}{p-1} \psi_{\mu}=0
$$

Theorem 3 of $\left[\operatorname{Ar} 1\right.$, p. 92], yields that $\psi$ is well defined in $\left\{\left(q^{\prime}, \theta^{\prime}\right): q^{\prime}>q_{0}^{\prime}\right\}$. To be able to define $\psi$ in $\Omega_{0}$ and $\omega\left(q_{0}\right)$ we must in general make a cut in each one of these domains in order to make them simply connected (see [Ar2, Theorem 1, p. 75]). However, from the $p$-Cauchy-Riemann equations (2) we see that the partial derivatives of $\psi$ can be continued smoothly across the cut. Since the coordinate change from $z=x+i y$ to $\zeta=q e^{i \theta}$ is smooth we then conclude that the systems (4) and (5) hold in all of $\omega\left(q_{0}\right)$.

\section{A representation theorem and some consequences}

\subsection{Analysis of $\varphi$ and $z(w)$.}

Theorem 2. Suppose $\varphi$ is $p$-harmonic in $\Omega=\{z: 0<|z|<1\} \quad(1<p<\infty)$ and that $\varphi$ has a pole of order $N-1$ at $z=0(N \geq 1)$. For $m \neq 0$ put

$$
\beta_{m}=\frac{1}{2}\left(\sqrt{(p-2)^{2}+4 \frac{m^{2}}{N^{2}}(p-1)} \pm(p-2)\right),
$$


where the + sign is chosen if $m \geq 1$ and the - sign if $m \leq-1 . \beta_{m}$ is positive for all $m$. For $-N+1 \leq m \leq-1, \beta_{m}$ is less than one and strictly decreasing in $m$, and for $m \geq 1, \beta_{m}$ is strictly increasing.

$$
\text { Define } Z_{m}(\varrho, \theta) \text { by }
$$

$$
Z_{m}(\varrho, \theta)= \begin{cases}\frac{i m e^{i N \theta}}{N\left(1-\beta_{m}\right)} \varrho^{N\left(1-\beta_{m}\right)}\left(\cos m\left(\theta-\theta_{m}\right)-i \frac{N \beta_{m}}{m} \sin m\left(\theta-\theta_{m}\right)\right) \\ \frac{e^{i N \theta}}{N(p-1)} \varrho^{N(p-1)} & \text { if }-N+1 \leq m \leq-1 \\ -\frac{i m e^{i N \theta}}{N\left(1+\beta_{m}\right)} \varrho^{N\left(1+\beta_{m}\right)}\left(\cos m\left(\theta-\theta_{m}\right)+i \frac{N \beta_{m}}{m} \sin m\left(\theta-\theta_{m}\right)\right) & \text { if } m \geq 1 .\end{cases}
$$

Then there exists a $\varrho_{0}$ such that $z(\omega)$ and $\varphi$ admits a parametric representation as follows:

a) There exist real sequences $\left\{A_{m}\right\}_{m=-N+1}^{\infty},\left\{\theta_{m}\right\}_{m=-N+1}^{\infty}$ and a real number $b_{0}$ such that $A_{-N+1} \neq 0, A_{m} \geq 0$ for $m \neq 0, A_{m} \varrho_{0}^{N \beta_{m}}=O\left(m^{-k}\right)$ for any positive integer $k, 0 \leq \theta_{m}<2 \pi$ and $\theta_{0}=0$. The formulas

$$
w=\varrho e^{i \theta} \quad(\varrho>0), \quad z(\varrho, \theta)=\sum_{m=-N+1}^{\infty} A_{m} Z_{m}(\varrho, \theta)
$$

then define a homeomorphism from $W_{0}=\left\{w:|w| \leq \varrho_{0}\right\}$ onto a neighbourhood $\Omega_{0}$ of $z=0$ and $z(\varrho, \theta) \in C^{\infty}\left(\left(0, \varrho_{0}\right] \times \mathbf{R}\right)$.

b) Let

$$
\varphi_{0}(\varrho)= \begin{cases}\frac{A_{0}}{N(p-2)} \varrho^{N(p-2)}+b_{0} & \text { if } p \neq 2 \\ A_{0} \log \varrho+b_{0} & \text { if } p=2 .\end{cases}
$$

For $0<\varrho \leq \varrho_{0}$ the value of $\varphi$ at $z=z(\varrho, \theta)$ is given by

(7) $\varphi=\sum_{m=-N+1}^{-1} \frac{A_{m}}{\varrho^{N \beta_{m}}} \sin m\left(\theta-\theta_{m}\right)+\varphi_{0}(\varrho)+\sum_{m=1}^{\infty} A_{m} \varrho^{N \beta_{m}} \sin m\left(\theta-\theta_{m}\right)$.

The series for $z$ and $\varphi$ converge uniformly for $0 \leq \varrho \leq \varrho_{0}$ and $0<\varrho \leq \varrho_{0}$, respectively. Finally, the value of $\varphi_{x}+i \varphi_{y}$ at $z=z(\varrho, \theta)$ is $\left(\varrho^{-1} e^{i \theta}\right)^{N}=\bar{w}^{-N}$.

Proof. In order to get a neighbourhood of $z=0$ in one-to-one correspondence with a neighbourhood of 0 in the modified hodograph plane we put $q=\varrho^{-1}$. We then have the following relations between the different coordinates:

$$
\sqrt[N]{\varphi_{x}+i \varphi_{y}}=\zeta=q e^{i \theta}=\frac{1}{\varrho} e^{i \theta}=\frac{1}{\bar{w}}, \quad \mu=-\log q=\log \varrho .
$$


$\Omega_{0}$ is now in one-to-one correspondence with the set $W_{0}=\left\{w: 0<|w| \leq \varrho_{0}\right.$ $\left.=q_{0}^{-1}\right\}$ and $z(w) \in C\left(\bar{W}_{0}\right) \cap C^{\infty}\left(W_{0}\right)$.

Let $\left\{\alpha_{m}(\varrho)\right\}_{-\infty}^{\infty}$ be the Fourier coefficients of the function $\varphi(\varrho, \cdot)$. Since $\varphi \in C^{\infty}\left(\left(0, \varrho_{0}\right] \times \mathbf{R}\right)$ and $\varphi(\varrho, \cdot)$ is periodic with period $2 \pi$ we have that

$$
\alpha_{m}(\varrho)=\frac{1}{2 \pi} \int_{0}^{2 \pi} \varphi(\varrho, \theta) e^{-i m \theta} d \theta
$$

is in $C^{\infty}\left(\left(0, \varrho_{0}\right]\right)$. To simplify the calculations we switch from $\varrho$ to $\mu$. We shall also abuse notation slightly and write

$$
\alpha_{m}(\mu)=\frac{1}{2 \pi} \int_{0}^{2 \pi} \varphi(\mu, \theta) e^{-i m \theta} d \theta .
$$

Now, let $m \geq 1$ and differentiate $\alpha_{m}(\mu)$ twice. From (5) we get

$$
\varphi_{\mu \mu}=N(p-2) \varphi_{\mu}-(p-1) \varphi_{\theta \theta}
$$

Thus,

$$
\begin{aligned}
\frac{d^{2}}{d \mu^{2}} \alpha_{m}(\mu) & =N(p-2) \frac{1}{2 \pi} \int_{0}^{2 \pi} \varphi_{\mu}(\mu, \theta) e^{-i m \theta} d \theta-(p-1) \frac{1}{2 \pi} \int_{0}^{2 \pi} \varphi_{\theta \theta}(\mu, \theta) e^{-i m \theta} d \theta \\
(8) & =N(p-2) \frac{d}{d \mu} \alpha_{m}(\mu)+m^{2}(p-1) \alpha_{m}(\mu) .
\end{aligned}
$$

Solving this equation and replacing $e^{\mu}$ with $\varrho$ yields

$$
\alpha_{m}(\varrho)=a_{m} \varrho^{N \beta_{m}}+a_{-m} \varrho^{-N \beta_{-m}}
$$

where

$$
\beta_{m}=\frac{1}{2}\left(\sqrt{(p-2)^{2}+4 \frac{m^{2}}{N^{2}}(p-1)} \pm(p-2)\right),
$$

with + if $m \geq 1$ and - if $m \leq-1$. Since $\varphi$ is real it follows that

$$
\alpha_{-m}(\varrho)=\overline{\alpha_{m}(\varrho)}=\overline{a_{m}} \varrho^{N \beta_{m}}+\overline{a_{-m}} \varrho^{-N \beta_{-m}} .
$$

The remaining term $\alpha_{0}(\mu)=\frac{1}{2 \pi} \int_{0}^{2 \pi} \varphi_{\mu \mu} d \theta$ is obtained in the same way by differentiating twice. Using (5) we get

$$
\begin{aligned}
\frac{d^{2}}{d \mu^{2}} \alpha_{0}(\mu) & =\frac{1}{2 \pi} \int_{0}^{2 \pi} \varphi_{\mu \mu} d \theta=N(p-2) \frac{d}{d \mu} \alpha_{0}(\mu)-(p-1) \frac{1}{2 \pi} \int_{0}^{2 \pi} \varphi_{\theta \theta} d \theta \\
& =N(p-2) \frac{d}{d \mu} \alpha_{0}(\mu)
\end{aligned}
$$


Solving this equation yields

$$
\alpha_{0}(\varrho)=\varphi_{0}(\varrho)= \begin{cases}\frac{A_{0}}{N(p-2)} \varrho^{N(p-2)}+b_{0} & \text { if } p \neq 2, \\ A_{0} \log \varrho+b_{0} & \text { if } p=2 .\end{cases}
$$

Before writing the series for $\varphi$ we derive the series for $z$ since this will lead us to conclude that some of the coefficients $a_{m}$ are zero.

Define

$$
F_{m}(\varrho)=\frac{1}{2 \pi} \int_{0}^{2 \pi} z(\varrho, \theta) e^{-i(N+m) \theta} d \theta
$$

i.e. $\left\{F_{m}(\varrho)\right\}_{-\infty}^{\infty}$ are the Fourier coefficients of the function $e^{-i N \cdot} z(\varrho, \cdot)$ which belongs to $C^{\infty}(\mathbf{R})$ for $0<\varrho \leq \varrho_{0}$. Since

$$
\frac{\partial(\varphi, \psi)}{\partial(x, y)}=|\nabla \varphi|^{p}>0
$$

we see that $\varphi$ and $\psi$ are valid as new coordinates in a neighbourhood of any point in $\Omega_{0}$. From [Ar1, p. 90] we have, locally, that

$$
\frac{\partial z}{\partial \varphi}=\frac{e^{i \theta^{\prime}}}{q^{\prime}} \quad \text { and } \quad \frac{\partial z}{\partial \psi}=\frac{i e^{i \theta^{\prime}}}{\left(q^{\prime}\right)^{p-1}}
$$

Since $q^{\prime}=q^{N}$ and $\theta^{\prime}=N \theta$ we get

$$
\frac{\partial z}{\partial \varphi}=\frac{e^{i N \theta}}{q^{N}} \quad \text { and } \quad \frac{\partial z}{\partial \psi}=\frac{i e^{i N \theta}}{q^{N(p-1)}} .
$$

The fact that these equations are only valid locally causes no problems since we can split the interval of integration in as many and as small parts as we need.

We proceed in the same manner as above and differentiate to obtain

$$
\begin{aligned}
\frac{d}{d \mu} F_{m}(\mu) & =\frac{1}{2 \pi} \int_{0}^{2 \pi}\left(\frac{\partial z}{\partial \varphi} \frac{\partial \varphi}{\partial \mu}+\frac{\partial z}{\partial \psi} \frac{\partial \psi}{\partial \mu}\right) e^{-i(N+m) \theta} d \theta \\
& =\frac{1}{2 \pi} \int_{0}^{2 \pi}\left(e^{N \mu} \varphi_{\mu}(\mu, \theta)+i e^{N(p-1) \mu} \psi_{\mu}(\mu, \theta)\right) e^{-i m \theta} d \theta
\end{aligned}
$$

From the Chaplygin system (4) we see that $\psi_{\mu}=-e^{-N(p-2) \mu} \varphi_{\theta}$, and thus for $m \geq 1$ we get

$$
\begin{aligned}
\frac{d}{d \mu} F_{m}(\mu) & =\frac{1}{2 \pi} \int_{0}^{2 \pi} e^{N \mu}\left(\varphi_{\mu}-i \varphi_{\theta}\right) e^{-i m \theta} d \theta=e^{N \mu}\left(\frac{d}{d \mu} \alpha_{m}(\mu)+m \alpha_{m}(\mu)\right) \\
& =a_{m}\left(m+N \beta_{m}\right) e^{N\left(1+\beta_{m}\right) \mu}+a_{-m}\left(m-N \beta_{-m}\right) e^{N\left(1-\beta_{-m}\right) \mu} \\
\frac{d}{d \mu} F_{-m}(\mu) & =e^{N \mu}\left(\frac{d}{d \mu} \alpha_{-m}(\mu)-m \alpha_{-m}(\mu)\right)=e^{N \mu}\left(\frac{d}{d \mu} \overline{\alpha_{m}(\mu)}-m \overline{\alpha_{m}(\mu)}\right) \\
& =\overline{a_{m}}\left(N \beta_{m}-m\right) e^{N\left(1+\beta_{m}\right) \mu}-\overline{a_{-m}}\left(N \beta_{-m}+m\right) e^{N\left(1-\beta_{-m}\right) \mu} \\
\text { (13) } & \text { (14) } \frac{d}{d \mu} F_{0}(\mu) \\
= & A_{0} e^{N(p-1) \mu}
\end{aligned}
$$


If $m=N$ then $\beta_{-N}=1$ and $\beta_{N}=p-1$. Hence, if we integrate (12), (13) and (14) and replace $e^{\mu}$ with $\varrho$ we obtain for $m \geq 1$

$$
\begin{gathered}
F_{m}(\varrho)=\left\{\begin{array}{cc}
a_{m} \frac{m+N \beta_{m}}{N\left(1+\beta_{m}\right)} \varrho^{N\left(1+\beta_{m}\right)}+ \\
+a_{-m} \frac{m-N \beta_{-m}}{N\left(1-\beta_{-m}\right)} \varrho^{N\left(1-\beta_{-m}\right)}+c_{m} & \text { if } m \neq N \\
a_{N} \varrho^{N p}+c_{N} & \text { if } m=N
\end{array}\right. \\
F_{-m}(\varrho)=\left\{\begin{array}{cc}
\overline{a_{m}} \frac{N \beta_{m}-m}{N\left(1+\beta_{m}\right)} \varrho^{N\left(1+\beta_{m}\right)}- & \text { if } m \neq N \\
-\overline{a_{-m}} \frac{m+N \beta_{-m}}{N\left(1-\beta_{-m}\right)} \varrho^{N\left(1-\beta_{-m}\right)}+c_{-m} & \text { if } m \\
\frac{\overline{a_{N}}(p-2)}{p} \varrho^{N p}-2 N a_{-N} \log \varrho+c_{-N} & \\
F_{0}(\varrho)=\frac{A_{0}}{N(p-1)} \varrho^{N(p-1)}+c_{0} .
\end{array}\right.
\end{gathered}
$$

It is clear that $\beta_{m}$ and $\beta_{-m}$ are increasing as functions of $m$. Since $\beta_{-N}=1$ it follows that $1-\beta_{-m}<0$ for $m \geq N+1$. This together with $\lim _{\varrho \rightarrow 0} z(\varrho, \theta)=0$ implies

$$
\lim _{\varrho \rightarrow 0}\left|F_{m}(\varrho)\right| \leq \lim _{\varrho \rightarrow 0} \frac{1}{2 \pi} \int_{0}^{2 \pi}|z(\varrho, \theta)| d \theta=0
$$

for every $m$. Hence $c_{m}=0$ for every $m$ and $a_{m}=0$ for $m \leq-N$.

We can now write the series for $\varphi$ in the following form.

$$
\begin{aligned}
\varphi(\varrho, \theta) & =\sum_{m=-\infty}^{\infty} \alpha_{m}(\varrho) e^{i m \theta} \\
& =\sum_{m=-N+1}^{-1} \frac{A_{m}}{\varrho^{N \beta_{m}}} \sin m\left(\theta-\theta_{m}\right)+\varphi_{0}(\varrho)+\sum_{m=1}^{\infty} A_{m} \varrho^{N \beta_{m}} \sin m\left(\theta-\theta_{m}\right)
\end{aligned}
$$

where $A_{m}=2\left|a_{m}\right|$ for $m \neq 1$ and $\theta_{m}$ depends on $a_{m}$. This proves (7).

Define $Z_{m}(\varrho, \theta)$ as in (6). For any $\varrho \leq \varrho_{0}$ we thus have

$$
z(\varrho, \theta)=\sum_{m=-\infty}^{\infty} F_{m}(\varrho) e^{i(N+m) \theta}=\sum_{m=-N+1}^{\infty} A_{m} Z_{m}(\varrho, \theta) .
$$


To finish the proof we need only show that $A_{-N+1} \neq 0$. Put

$$
\gamma_{m}= \begin{cases}N\left(1-\beta_{m}\right) & \text { if }-N+1 \leq m \leq-1, \\ N(p-1) & \text { if } m=0 \\ N\left(1+\beta_{m}\right) & \text { if } m \geq 1\end{cases}
$$

Looking at the formula for $\beta_{m}$ we easily see that

$$
\lim _{m \rightarrow \infty} \frac{N \beta_{m}}{m}=\sqrt{p-1}, \quad \lim _{m \rightarrow \infty} N \beta_{m}-m \sqrt{p-1}=\frac{N(p-2)}{2} .
$$

The sequence $\left\{\gamma_{m}\right\}_{-N+1}^{\infty}$ is positive and strictly increasing. This together with $(19)$ then implies that there exist positive linear functions $f_{1}(m)$ and $f_{2}(m)$ such that

$$
f_{1}(m) \leq \gamma_{m} \leq f_{2}(m) \quad \text { for every } m \geq-N+1 .
$$

Thus there exist positive constants $c$ and $d$, independent of $m$ and $\theta$ such that

$$
c \varrho^{\gamma_{m}} \leq\left|Z_{m}(\varrho, \theta)\right| \leq d \varrho^{\gamma_{m}} \quad \text { for every } m \geq-N+1 .
$$

Let $M \geq-N+1$ be the smallest integer such that $A_{M} \neq 0$. Since $z(\varrho, \cdot) \in$ $C^{\infty}(\mathbf{R})$ for $0<\varrho \leq \varrho_{0}$ we get that $A_{m} \varrho_{0}^{\gamma_{m}}=O\left(m^{-k}\right)$ for any $k \geq 1$. This together with (20) and (21) implies

$$
\begin{aligned}
\sum_{M+1}^{\infty} A_{m}\left|Z_{m}(\varrho, \theta)\right| & \leq C \sum_{M+1}^{\infty} A_{m} \varrho_{0}^{\gamma_{m}}\left(\frac{\varrho}{\varrho_{0}}\right)^{\gamma_{m}} \\
& =C\left(\frac{\varrho}{\varrho_{0}}\right)^{\gamma_{M+1}} \sum_{M+1}^{\infty} A_{m} \varrho_{0}^{\gamma_{m}}\left(\frac{\varrho}{\varrho_{0}}\right)^{\gamma_{m}-\gamma_{M+1}} \\
& \leq C\left(\frac{\varrho}{\varrho_{0}}\right)^{\gamma_{M+1}} \leq C \varrho^{\gamma_{M+1}} \leq \frac{1}{2} A_{M}\left|Z_{M}(\varrho, \theta)\right| .
\end{aligned}
$$

Thus, we immediately get

$$
\left|z(\varrho, \theta)-A_{M} Z_{M}(\varrho, \theta)\right| \leq \frac{1}{2}\left|A_{M} Z_{M}(\varrho, \theta)\right|
$$

if $\varrho>0$ is sufficiently small. Take such a $\varrho$ and let $\theta$ increase from $\theta_{M}$ to $\theta_{M}+2 \pi$. By the above reasoning and inspection of the formula for $Z_{M}(\varrho, \theta)$ we see that the change in argument is

$$
\arg z\left(\varrho, \theta_{M}+2 \pi\right)-\arg z\left(\varrho, \theta_{M}\right)=\arg Z_{M}(\varrho, 2 \pi)-\arg Z_{M}(\varrho, 0)=2 \pi(N+M) .
$$

Since we know that the difference should be + or $-2 \pi$ we get $M=-N \pm 1$. Since $A_{-N-1}=0$ we get

$$
A_{-N+1} \neq 0
$$

This completes the proof.

Remark. In $[\mathrm{M}], \mathrm{J}$. Manfredi obtained the same series for $z(\varrho, \theta)$ using a different variant of the hodograph method. The series in $[M]$ is obtained in the same way as here, i.e. by studying an ordinary differential equation for the Fourier coefficients. 
3.2. Analysis of the stream function $\psi$. Let $\Omega$ and $\Omega_{0}$ be as in Theorem 2 and define $\widetilde{\Omega}$ as above. From the previous sections we know that $\varphi$ has a $p^{\prime}$-harmonic stream function $\psi$ in $\widetilde{\Omega}$. Further, $\varphi$ and $\psi$ satisfy the $p$-CauchyRiemann equations (2) in $\Omega$. Hence $\nabla \psi$ is smooth in $\Omega$.

Let $\Lambda$ be a positively oriented simple closed curve around $z=0$ such that $\Lambda$ is contained in $\Omega_{0}$. It is clear that $\psi$ is single-valued in $\Omega_{0}$ if and only if

$$
\int_{\Lambda} d \psi=\int_{\Lambda} \psi_{x} d x+\psi_{y} d y=0
$$

for every such $\Lambda$.

Theorem 3. Let $\varphi$ satisfy the conditions in Theorem 2 and let $\psi$ be a stream function of $\varphi$ in $\widetilde{\Omega}$. Let $\Lambda$ be as above. Then

$$
\int_{\Lambda} d \psi=\frac{2 \pi A_{0}}{p-1}
$$

i.e. $\psi$ is single-valued in $\Omega_{0}$ if and only if $N \geq 2$ and $A_{0}=0$.

Proof. Let $0<\varrho^{*} \leq \varrho_{0}$ and put $\Lambda=\left\{z:|\nabla \varphi(z)|=1 /\left(\varrho^{*}\right)^{N}\right\}$ with positive orientation. Then $\Lambda$ satisfies the above conditions and

$$
\int_{\Lambda} d \psi=\int_{\substack{0 \leq \theta \leq 2 \pi \\ \varrho=\varrho^{*}}} \psi_{\varrho}\left(\varrho^{*}, \theta\right) d \varrho+\psi_{\theta}\left(\varrho^{*}, \theta\right) d \theta=\int_{0}^{2 \pi} \psi_{\theta}\left(\varrho^{*}, \theta\right) d \theta .
$$

From the Chaplygin system (4) we get

$$
\psi_{\theta}=\frac{\varrho^{1-N(p-2)}}{p-1} \varphi_{\varrho}
$$

Hence,

$$
\int_{\Lambda} d \psi=\frac{\left(\varrho^{*}\right)^{1-N(p-2)}}{p-1} \int_{0}^{2 \pi} \varphi_{\varrho}\left(\varrho^{*}, \theta\right) d \theta=\frac{2 \pi\left(\varrho^{*}\right)^{1-N(p-2)}}{p-1} \frac{d \alpha_{0}}{d \varrho}\left(\varrho^{*}\right) .
$$

$(d / d \varrho) \alpha_{0}(\varrho)=A_{0} \varrho^{N(p-2)-1}$ yields $\int_{\Lambda} d \psi=\left(2 \pi A_{0}\right) /(p-1) . \psi$ is single-valued if and only if $\int_{\Lambda} d \psi=0$, that is if and only if $A_{0}=0$. Since $A_{0} \neq 0$ if $N=1$ the statement follows.

We will now derive an expression for $\psi(\varrho, \theta)$. It is clear that the series for $\varphi$ can be differentiated term by term as many times as we please. Thus, if we insert the series for $\varphi$ in (4) and solve the system for $\psi$ we obtain

$$
\begin{gathered}
\psi(\varrho, \theta)=\sum_{m=-N+1}^{-1} \frac{m A_{m}}{N \beta_{-m} \varrho^{N \beta_{-m}}} \cos m\left(\theta-\theta_{m}\right)+\frac{A_{0}}{p-1} \theta \\
-\sum_{m=1}^{\infty} \frac{m A_{m}}{N \beta_{-m}} \varrho^{N \beta_{-m}} \cos m\left(\theta-\theta_{m}\right) .
\end{gathered}
$$

We can now make the following additions to part b) of Theorem 2: 
Theorem 4. Assumptions and part a) as in Theorem 2 .

b) If we cut $\Omega_{0}$ along the curve $\left\{z: z=z(\varrho, 0), 0 \leq \varrho \leq \varrho_{0}\right\}$ then, in the resulting domain $\widetilde{\Omega}, \psi$ can be represented by a series of the form (24). The series (24) is uniformly convergent for $0<\varrho \leq \varrho_{0}$. Further, the value of $\psi_{x}+i \psi_{y}$ at $z=z(\varrho, \theta)$ is $i\left(\varrho^{1-p} e^{i \theta}\right)^{N}$.

Remark. In the case where $\psi$ is single-valued it has a pole at $z=0$ of the same order as the pole of $\varphi$. This is seen by substituting $p^{\prime} /\left(p^{\prime}-1\right)$ for $p$ and $\left(\varrho^{\prime}\right)^{1 /\left(p^{\prime}-1\right)}$ for $\varrho$ in $(24)$.

We can also derive the following converse of the extended representation theorem.

Theorem 5. Let $N \geq 1,1<p<\infty$ and $\left\{A_{m}\right\}_{m=-N+1}^{\infty} \subset \mathbf{R}$ be such that $A_{-N+1} \neq 0$ and $\left\{A_{m} \varrho^{N \beta_{m}}\right\}_{m=-N+1}^{\infty}$ is bounded for some $\varrho>0$. Let $\left\{\theta_{m}\right\}_{m=-N+1}^{\infty} \subset[0,2 \pi)$ where $\theta_{0}=0$.

Define $Z_{m}(\varrho, \theta)$ as in (6) and put

$$
z(\varrho, \theta)=\sum_{m=-N+1}^{\infty} A_{m} Z_{m}(\varrho, \theta) .
$$

Then there is a $\varrho_{0}>0$ such that for $0 \leq \varrho \leq \varrho_{0}$, the mapping $z=z(\varrho, \theta)$ is a homeomorphism from $W_{0}=\left\{w=\varrho e^{i \theta}: 0 \leq \varrho \leq \varrho_{0}\right\}$ onto a neighbourhood $\Omega_{0}$ of $z=0$ and $z(\varrho, \theta) \in C^{\infty}\left(W_{0} \backslash\{0\}\right)$.

Put $\Gamma=\left\{z: z=z(\varrho, 0), \varrho \leq \varrho_{0}\right\}$. Then the formulas (7) and (24) define $\varphi$ and $\psi$ implicitly as functions of $z$ in $\Omega_{0} \backslash\{0\}$ and $\Omega_{0} \backslash \Gamma$, respectively. Further, $\varphi$ is $p$-harmonic in $\Omega_{0} \backslash\{0\}$ with a pole of order $N-1$ at $z=0$ and $\psi$ is a stream function of $\varphi$. If $A_{0}=0$ then $\psi$ is single-valued, i.e. defined in $\Omega_{0} \backslash\{0\}$.

Proof. In [Ar2, p. 86-89] a similar mapping $z(\varrho, \theta)$ is thoroughly analyzed for the case that $\varphi$ has a critical point at $z=0$, i.e. $\nabla \varphi(0)=0$. There it is shown that the mapping properties of $z(\varrho, \theta)$ are determined by the leading term in the series. The same proof also applies here (with obvious modifications). Thus $z=z(\varrho, \theta)$ is a homeomorphism with the desired properties.

If we put $q^{\prime}=\varrho^{-N}$ and $\theta^{\prime}=N \theta$ then Theorem 3 of [Ar1] applies locally in a neighbourhood of any point in $W_{0}$ with $\varrho>0$ and $0<\theta<2 \pi$. Hence $\varphi$ and $\psi$ have the stated properties. $\square$

3.3. Singular expansions of $\varphi$ and $\psi$. Suppose that $\varphi$ is $p$-harmonic in $\Omega=\{z: 0<|z|<1\}, 1<p<\infty, p \neq 2$ and that $\psi$ is a stream function for $\varphi$ on $\Omega \backslash\left\{z: \varphi_{x}(z) \geq 0, \varphi_{y}(z)=0\right\}$. Further, suppose that $\varphi$ has a pole of order $N-1$ at $z=0(N \geq 1)$ and put $z=r e^{i \phi}$. It is then possible to derive singular expansions of the following type:

$$
\varphi\left(r e^{i \phi}\right)=r^{k_{N}} f(\phi)+O\left(r^{k_{N}+\delta_{N}}\right), \quad \psi\left(r e^{i \phi}\right)=r^{l_{N}} g(\phi)+O\left(r^{l_{N}+\gamma_{N}}\right) .
$$


The functions $r^{k_{N}} f(\phi)$ and $r^{l_{N}} g(\phi)$ are $p$-and $p^{\prime}$-harmonic respectively and generated implicitly by the leading terms in the series for $\varphi$ and $\psi$, respectively, together with the leading term in the series for $z$.

It can then be shown that

$$
k_{N}=\left\{\begin{array}{ll}
-\frac{\beta_{-N+1}}{1-\beta_{-N+1}} & \text { if } N \geq 2 \\
\frac{p-2}{p-1} & \text { if } N=1,
\end{array} \quad k_{N}+\delta_{N}= \begin{cases}-\frac{\beta_{-N+2}}{1-\beta_{-N+1}} & \text { if } N \geq 3 \\
\frac{p-2}{1-\beta_{-1}} & \text { if } N=2 \\
1 & \text { if } N=1\end{cases}\right.
$$

$l_{N}=\left\{\begin{array}{ll}-\frac{\beta_{N-1}}{1-\beta_{-N+1}} & \text { if } N \geq 2, \\ 0 & \text { if } N=1,\end{array} \quad\right.$ and $\quad l_{N}+\gamma_{N}= \begin{cases}-\frac{\beta_{N-2}}{1-\beta_{-N+1}} & \text { if } N \geq 3, \\ 0 & \text { if } N=2, \\ \frac{1}{p-1} & \text { if } N=1 .\end{cases}$

Further, $l_{N}-1=(p-1)\left(k_{N}-1\right)$.

The proof of this is analogous to the proof of Theorem 6 in [Ar2] and it can be found in [J, p. 22-25].

Remark. J. Manfredi has in [M], under the same assumptions, obtained a singular expansion for $\varphi$. In the cases $N \geq 3$ and $N=1, p>2$, the result is exactly the same as ours. The case $N=2,1<p<2$ is not treated in [M] and in the remaining cases, the exponent $k_{N}+\delta_{N}$ given here is larger.

\section{Classification of isolated singularities}

The complex gradient $\varphi_{x}-i \varphi_{y}$, of any $p$-harmonic function has a representation $h \circ \chi$ where $h$ is analytic and $\chi$ is quasi-conformal. Since isolated singularities of quasi-conformal mappings are removable (see $[\mathrm{V}, \mathrm{p} .52]$ ) we see that $\varphi$ has an isolated singularity at $z_{0}$ if and only if $h$ has an isolated singularity at $\chi\left(z_{0}\right)$. This leads to a classification of the singularities as removable, poles and essential singularities.

Let $\Omega=\{z: 0<|z|<1\}$ and suppose $\varphi$ is $p$-harmonic in $\Omega(1<p<\infty)$. If $h$ has a removable singularity at $\chi(0)$ it is easy to show that

$$
\int_{\mathbf{R}^{2}}|\nabla \varphi|^{p-2} \nabla \varphi \cdot \nabla \eta d x d y=0 \quad \text { for all } \eta \in C_{0}^{\infty}(\Omega \cup\{0\}),
$$

i.e. $\varphi$ is $p$-harmonic in $\Omega \cup\{0\}$. We further say that $\varphi$ has an essential singularity at $z_{0}$ if $h$ has an essential singularity at $\chi\left(z_{0}\right)$.

The type of singularity of $h$ at $\chi(0)$ is independent of the choice of representation, $\varphi_{x}-i \varphi_{y}=h \circ \chi$ (see $\left.[\mathrm{R}]\right)$. From Theorem 2 we can now derive criteria for the different types of singularities stated directly in terms of $\varphi$. 
Remark. The following results are well known in the case $p=2$. In order to avoid unnecessary technical and typographical complications we exclude that case here. Although, if the calculations were to be carried out the result would be the same as the classical.

Theorem 6. Suppose that $\varphi$ is $p$-harmonic in $\Omega, 1<p<\infty$ and $p \neq 2$. Then $z=0$ is either a removable singularity, a pole of order $N-1 \geq 0$ or an essential singularity. The type of singularity is determined by the following criteria:

1. $z=0$ is a removable singularity if and only if

a) there exists a constant $A$ such that $\lim _{z \rightarrow 0} \varphi(z)=A, 1<p<2$,

b) $\lim _{z \rightarrow 0}|z|^{(2-p) /(p-1)}|\varphi(z)-A|=0$ for some constant $A, p>2$.

In both cases, chosing $\varphi(0)=A$ makes $\varphi$ regular.

2. $z=0$ is a pole of order 0 (singularity of fundamental solution type) if and only if

a) $\lim _{z \rightarrow 0}|z|^{(2-p) /(p-1)}|\varphi(z)|=C>0,1<p<2$,

b) $\lim _{z \rightarrow 0}|z|^{(2-p) /(p-1)}|\varphi(z)-A|=C>0$ for some constant $A, p>2$. Choosing $\varphi(0)=A$ makes $\varphi$ continuous.

3. $z=0$ is a pole of order $N-1 \geq 1$ if and only if

$$
\limsup _{z \rightarrow 0}|z|^{\gamma_{N}}|\varphi(z)|=C, \quad 0<C<\infty, \text { where } \gamma_{N}=\frac{\beta_{-N+1}}{1-\beta_{-N+1}} .
$$

This can occur for at most one value of $N$.

4. $z=0$ is an essential singularity if and only if

$$
\limsup _{z \rightarrow 0}|z|^{\alpha}|\varphi(z)|=\infty \quad \text { for all } \alpha \in \mathbf{R} .
$$

To prove this we need the following lemma ([M], Lemma 5).

Lemma 7 (Manfredi). Suppose that $\varphi$ is $p$-harmonic $(1<p<\infty)$ in $\left\{x \in \mathbf{R}^{2}: 0<|x|<1\right\}$. Further, suppose that the singularity at $x=0$ is not removable and that

$$
|\varphi(x)| \leq A|x|^{\beta} \quad \text { for } 0<|x| \leq 1 \quad \text { and some } \beta \leq \frac{p-2}{p-1} .
$$

Then there is a $C_{0}$ such that

$$
|\nabla \varphi(x)| \leq C_{0}|x|^{\beta-1} \quad \text { for } 0<|x| \leq \frac{1}{2} .
$$

Proof of Theorem 6: Case 1. Suppose $\lim _{z \rightarrow 0} \varphi(z)=A$. Put $\varphi(0)=A$. Then there is a constant $B$ such that $\varphi(z)+B>0$ for $|z| \leq \frac{1}{2}$. Put $\widetilde{\varphi}(w)=$ $\varphi\left(\frac{1}{2} w\right)+B$. Then $\widetilde{\varphi}(w)$ is positive and $p$-harmonic for $0<|w|<1$. Classical 
results (see [S2, Theorem 1, p. 79]) say that in this case either the singularity is removable or there exist positive constants $C_{1}, C_{2}$ such that

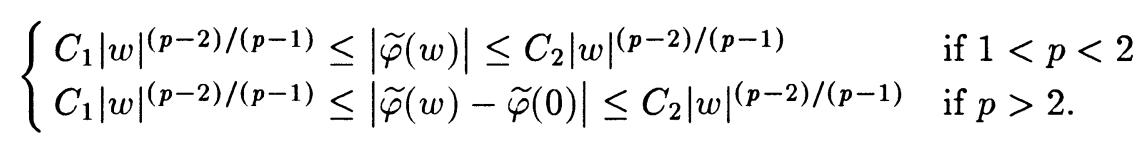

Since $C_{1}>0$ the singularity is removable.

Suppose $z=0$ is a removable singularity. We have that $\varphi \in C_{\text {loc }}^{1, \alpha}(\Omega \cup\{0\})$ by [L2]. Thus $\varphi$ is continuous at $z=0$ if $1<p<2$. If $p>2$, define $\widetilde{\varphi}(w)$ as above. Again using [S2, Theorem 1, p. 79] we get that $|\widetilde{\varphi}(w)-\widetilde{\varphi}(0)|$ tends to zero faster than $|w|^{(p-2) /(p-1)}$. Thus b) holds.

Cases 2 and 3. If $z=0$ is a pole of order $N-1 \geq 0$ then we are in the case treated in Theorem 2 which also provides the stated exponents of $|z|$.

Suppose one of the limits in question in 2 or 3 exists. Then Lemma 7 applies. Hence $\varphi$ can not have an essential singularity, because if $h$ has an essential singularity, then $|\chi(z)|^{\alpha}|h \circ \chi(z)|$ is unbounded for every $\alpha \in \mathbf{R}$. Since $K$-quasiconformal mappings are Hölder continuous with exponent $K^{-1}$ (see [L-V, Satz 4.3$, p. 73$])$ it follows that

$$
|z|^{\alpha}|h \circ \chi(z)|=|z|^{\alpha}|\nabla \varphi(z)|
$$

is unbounded for every $\alpha$, which contradicts the lemma. From 1 we get that the singularity is not removable. Thus the only possibility is a pole or singularity of fundamental solution type. Since $\gamma(N)>(2-p) /(p-1)$ and strictly increasing as a function of $N$ it follows by Theorem 2 that the order of the pole is $N-1$.

Case 4. Suppose $z=0$ is an essential singularity. Then by definition, $h$ in the representation $\varphi_{x}-i \varphi_{y}=h \circ \chi$ has an essential singularity. By the preceding argument this is equivalent to saying that

$$
|z|^{\alpha}|h \circ \chi(z)|=|z|^{\alpha}|\nabla \varphi(z)|
$$

is unbounded for every $\alpha$. This completes the proof. $\square$

Corollary. If $\int_{\Omega}|\nabla \varphi|^{\alpha} d x<\infty$ for some $\alpha>0$, then the singularity is not essential.

Proof. By Lemma 6 in $[\mathrm{M}]$ we have that there exists $\lambda>0$ and a constant $C$ such that

$$
|\varphi(z)| \leq C|z|^{-\lambda} \text {. }
$$

Thus, by Theorem 6 the singularity is not essential. $\square$ 


\section{References}

[Ar1] Aronsson, G.: On certain $p$-harmonic functions in the plane. - Manuscripta Math. 61, 1988, 79-101.

[Ar2] ARonsson, G.: Representation of a $p$-harmonic function near a critical point in the plane. - Manuscripta Math. 66, 1989, 73-95.

[A-L] Aronsson, G., and P. Lindquist: On $p$-harmonic functions in the plane and their stream functions. - J. Differential Equations 74, 1988, 157-178.

[B-I] BojARSKI, B., and T. IWANIEC: $p$-harmonic equation and quasiregular mappings. Preprint SFB 72, 617, 1983, Universität Bonn.

[H] HopF, E.: Über den funktionalen, insbesondere den analytischen Charakter, der Lösungen elliptischer Differentialgleichungen zweiter Ordnung. - Math. Z. 34:2, 1931/1932, 194233.

[J] JANFALK, U.: Representation of a $p$-harmonic function near an isolated singularity in the plane. - Licentiate Thesis, 1991:13, Linköping University.

[L-V] Lehto, O., and K.I. VirTanen: Quasikonforme Abbildungen. - Springer-Verlag, Berlin, 1965.

[L1] LEwIs, J.: Capacitary functions in convex rings. - Arch. Rational Mech. Anal. 66, 1977, 201-224.

[L2] LEwIS, J.: Regularity of the derivatives of certain degenerate elliptic equations. - Indiana Univ. Math. J. 32, 1983, 849-858.

[M] MANFredi, J.: Isolated singularities of $p$-harmonic functions in the plane. - SIAM J. Math. Anal. 22, 1991, 424-439.

[R] RENELT, H.: Elliptic systems and quasiconformal mappings. - Teubner, Leipzig, 1982 (English edition: John Wiley \& Sons, 1988).

[S1] SERrin, J.: Local behaviour of solutions of quasi-linear equations. - Acta Math. 111, 1964, $247-302$.

[S2] Serrin, J.: Singularities of solutions of nonlinear equations. - Proc. Sympos. Appl. Math. XVII, 1965, 68-88.

[V] VÄIsÄLÄ, J.: Lectures on $n$-dimensional quasiconformal mappings. - Springer-Verlag, Berlin, 1971. 